\title{
Analysis of Energy Consumption in Direct Transmission and Multi-hop Transmission for Wireless Sensor Networks
}

\author{
Jin Wang, Yu Niu, Jinsung Cho, Sungyoung Lee* \\ Department of Computer Engineering, \\ Kyung Hee University, Korea \\ \{wangjin,niuyu,sylee\}@oslab.khu.ac.kr \\ \{chojs\}@khu.ac.kr
}

\begin{abstract}
In this paper, we primary focus on prolonging the network lifetime of Wireless Sensor Networks (WSN), since the small, portable batteries integrated into the sensor chips can not be re-charged easily from an economical point of view. We first made a further analysis about the relationship between energy consumption and hop number. Then, an optimal hop number is deduced for minimizing the energy consumption during the multi-hop transmission. The importance of hop number to the energy consumption is usually neglected by many routing protocols. In fact, a considerable amount of energy can be saved if the relationship between hop number and energy consumption is carefully studied. After further analysis about the energy consumption of different transmission manner as well as design parameters, we presented our judging criterion of transmission manner. Also, an energy efficient routing scenario is presented with diagram so as to illustrate how the network lifetime can be prolonged.
\end{abstract}

\section{Introduction}

Wireless Sensor Networks (WSN) are composed of hundreds or thousands of tiny and inexpensive sensor nodes, which can monitor the surrounding environment through sensing, data processing and communication. The WSN have a variety of applications, such as military surveillance, industry monitoring, mass vehicle control and smart home etc [1].

\footnotetext{
* Professor Sungyoung Lee is the corresponding author.
}

One of the challenges of the successful WSN application is the energy consumption problem. It can be further divided into three sub-components, namely: sensing, processing and communication part. In this paper, we just focus on the energy consumption during communication since it plays a dominating role among three of them.

Currently, numerous works have been done to improve the routing performance in the network layer of WSN. [9] presents a taxonomy about most of the routing protocols for WSN and it categorizes them into three main classes, which are Data-centric [2, 3], Hierarchical [4, 5, 6] and Location-based [7, 8].

Data aggregation, also known as data fusion, is an important technique adopted by the data-centric routing protocols [2, 3]. It can reduce the energy consumption during communication process due to the fact that many nearby sensor nodes might sense and collect similar information. Consequently, there is more or less similarity among those collected sensor data. Through this method, both the size and the number of transmission can be reduced largely. However, the computational complexity will also increase since data aggregation method is introduced herein.

Hierarchical routing protocols $[4,5,6]$ have gained quite amount of attention in recent years. The key idea is that the whole network can be further divided into smaller areas called clusters. In each cluster, there is a cluster head which functions like a Base Station (BS). Within each cluster, each node simply communicates with the cluster head within a short range. The cluster heads communicate with each other to transmit their collected data to the remote BS. In this way, resources like spectrum or channel can be more efficiently utilized and load can get more balanced through the rotation of cluster head. Also, data aggregation can be 
done by the cluster head in a more efficient way. More importantly, the energy consumption can be greatly reduced since the communication range is largely reduced and the ordinary sensor nodes within one cluster can be put into sleep state according to a Time Division Multiple Access (TDMA) schedule, which is sent by cluster head. The disadvantage is that the clustering algorithms need to be carefully designed so that other performance parameters, such as packet delivery ratio, latency, might not get deteriorated.

In the location-based routing protocols [7, 8], sensor location information is required. So, either GPS devices need to be installed or some complex algorithms need to be devised to estimate the distance between each communication pair. For example, some complex signal attenuation algorithms need to be carefully designed. Also, the GPS devices consume a huge amount of energy. So, this kind of routing protocols may be useful under some special applications where the energy consumption is not the primary concern.

Unlike the traditional routing protocols, where the hop number plays a secondary role and the influence of hop number to energy consumption is mostly neglected, our method mainly focuses on the relationship between them in this paper.

Our contribution in this paper lies in the following three aspects. First, we made a comprehensive analysis and comparison about the energy model. The influence of design parameters to the energy consumption model is carefully studied with figurative and tabulated description. Second, we deduce an optimal hop number so that the energy consumption is minimized during multi-hop transmission process, given the fixed distance from source node to BS as well as some design parameters (hardware parameter). Finally, we present our judging criterion of selecting transmission manner (direct transmission or multi-hop transmission) to prolong the network lifetime.

The rest of the paper is organized as follows. In section 2, we present some background knowledge, such as the network model, basic assumptions, energy model as well as the problem statement. An optimal hop number is deduced for minimizing the energy consumption after the introduction of Lemma 1 in Section 3. In Section 4, we present our further study about the energy model. Different transmission manner as well as design parameters are studied and a transmission manner selection criterion is presented. In Section 5, an energy efficient routing scenario is provided and Section 6 concludes this paper and presents our future work.

\section{Background Knowledge}

\subsection{Network Model}

Table 1 lists some of the network parameters and their definitions, which are used in a Wireless Sensor Network (also in this paper).

Table 1. Network parameters

\begin{tabular}{|c|l|c|}
\hline Parameter & \multicolumn{1}{|c|}{ Definition } & Unit \\
\hline$[\mathrm{X}, \mathrm{Y}]$ & Network range & $\mathrm{m}^{2}$ \\
\hline $\mathrm{N}$ & Total number of nodes & $(1 \leq i \leq N)$ \\
\hline$n_{i}$ & The ith node & $\mathrm{m}$ \\
\hline $\mathrm{R}$ & $\begin{array}{l}\text { Transmission range of } \\
\text { each node }\end{array}$ & $\mathrm{m}$ \\
\hline$d_{i j}$ & Distance from $n_{i}$ to $n_{j}$ & $\mathrm{~J}$ \\
\hline$E_{I}$ & Initial energy for all nodes & $\mathrm{J}$ \\
\hline$e_{i}$ & Remaining energy of $n_{i}$ & \\
\hline
\end{tabular}

\subsection{Basic Assumptions}

i) All sensor nodes are assumed to be stationary and homogenous;

ii) The energy consumption of sensing and processing is not considered here;

iii) For one time, there is only one node transmitting the collected data to the BS;

iv) Only BS has location knowledge about all nodes.

Unlike some of the recent work, there is no sensor node which is supervisor to the others so that it can be continuously used as special relay node or cluster head. Again, due to the fact that communication process consumes much more energy than sensing and processing process, we just focus on the former part here. Also, we do not consider data aggregation here. The data transmitted by each individual sensor node can be considered as a raw data or an aggregated data under specific context.

\subsection{Energy Model}

A commonly used energy model is known as first order radio model $[4,5]$. Table 2 gives the related parameters and their definitions. Also, we assume an $d^{2}$ energy loss during transmission process and let $E_{\text {Tx-elec }}=E_{R x \text {-elec }}=E_{\text {elec }}, E_{T x-\text { amp }}=\varepsilon_{\text {amp }}$. So, to 
transmit a $l$-bits message over a distance $d$, the radio expends:

$$
\begin{aligned}
E_{T x}(l, d) & =E_{\text {Tx-elec }}(l)+E_{T x \text {-amp }}(l, d) \\
& =l^{*} E_{\text {elec }}+l^{*} \varepsilon_{\text {amp }} * d^{2}
\end{aligned}
$$

and to receive this message, the radio expends:

$$
E_{R x}(l)=E_{R x-\text { elec }}(l)=l^{*} E_{\text {elec }}
$$

and to forward this message by an intermediate node, the radio expends:

$$
\begin{aligned}
E_{F x}(l, d) & =E_{T x}(l, d)+E_{R x}(l) \\
& =2 l * E_{\text {elec }}+l^{*} \varepsilon_{\text {amp }} * d^{2}
\end{aligned}
$$

Table 2. Radio parameters

\begin{tabular}{|c|l|c|}
\hline Parameter & Definition & Unit \\
\hline$E_{\text {elec }}$ & $\begin{array}{l}\text { Energy dissipation } \\
\text { rate to run the radio }\end{array}$ & $50 \mathrm{~nJ} / \mathrm{bit}$ \\
\hline$\varepsilon_{\text {amp }}$ & $\begin{array}{l}\text { Energy dissipation } \\
\text { rate to run transmit } \\
\text { amplifier }\end{array}$ & $100 \mathrm{pJ} / \mathrm{bit} / \mathrm{m}^{2}$ \\
\hline$l$ & Data length & $\mathrm{bit}$ \\
\hline$d$ & $\begin{array}{l}\text { Node transmission } \\
\text { range }\end{array}$ & $\mathrm{m}$ \\
\hline
\end{tabular}

\subsection{Problem Statement}

Now, let us consider the following situation. Given a randomly deployed sensor network and one source node has some data to transmit to the remote BS:

1) Whether to transmit the data directly (single hop) or through multi-hop?

2) If through multi-hop, is there an optimal hop number so that the energy consumption can get minimized?

From Section 3 to Section 5, we will analyze these two problems from both theoretical and numerical aspects.

\section{Preliminary Mathematical Analysis of Energy Model}

\subsection{Direct Transmission or Multi-hop Transmission}

Inspired by the excellent work in $[4,8]$, we first introduce Lemma 1 which is listed and proven in [4]. In a linear network shown in Figure 1, where the distance between each node is $r$ and there are $n$ nodes during the communication session.

Lemma 1: Direct transmission consumes less energy than multi-hop transmission if:

$$
\frac{E_{\text {elec }}}{\varepsilon_{\text {amp }}}>\frac{r^{2} * n}{2}
$$

Since sensor nodes are stationary, the distance between source node and BS is fixed and it can be represented as $d=n * r$ according to Figure 1. Again, formula (4) can be re-formulated as:

$$
\frac{E_{\text {elec }}}{\varepsilon_{\text {amp }}}>\frac{r^{2} * n}{2}=\frac{d^{2}}{2 * n}
$$

According to the parameter values in Table 2, we can get $n>\frac{d^{2}}{1000}$.

In the real network environment, since the BS is usually far away from the sensed area $(d>100)$, so it is possible formula (4) holds true. Let:

$$
n_{e}=\frac{\varepsilon_{\text {amp }} * d^{2}}{2 * E_{\text {elec }}}
$$

when direct transmission consumes the same energy as multi-hop transmission.

$$
\text { n nodes }
$$

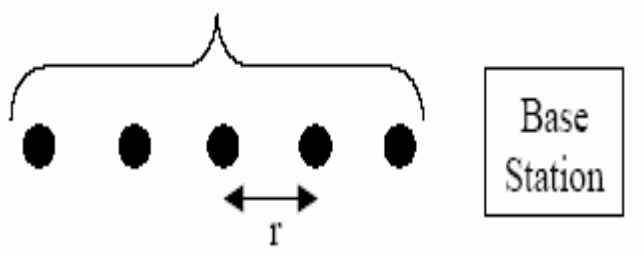

Figure 1. Simple linear network

\subsection{Optimal Hop Number}

For the direct transmission, the energy consumption will be (suppose $l=1$ bit):

$$
E_{1}=E_{\text {elec }}+\varepsilon_{\text {amp }} * d^{2}
$$

For the multi-hop transmission, the energy consumption will be:

$$
\begin{aligned}
& E_{2}(n)=E_{\text {elec }}+\varepsilon_{\text {amp }} * r^{2}+(n-1)\left(2 * E_{\text {elec }}+\right. \\
& \left.\varepsilon_{\text {amp }} * r^{2}\right)=2 n * E_{\text {elec }}+\frac{\varepsilon_{\text {amp }} * d^{2}}{n}-E_{\text {elec }}
\end{aligned}
$$

It is easy to prove that $E_{2}$ has a minimum value $\operatorname{Min} .\left(E_{2}\right)=\left(4 n^{*}-1\right) E_{\text {elec }}$ when:

$$
n=n^{*}=\sqrt{\frac{\varepsilon_{\text {amp }}}{2 * E_{\text {elec }}}} * d
$$


Here, $n^{*}$ is the optimal hop number during multi-hop transmission. Recall formula (5), we can draw another conclusion which is $n^{*}=\sqrt{n_{e}}$.

Since $n_{e}$ is obtained when direct communication consumes the same amount of energy as multi-hop transmission, we can easily prove $E_{2}(1)=E_{2}\left(n_{e}\right)$ $\geq E_{2}\left(n^{*}\right)$.

\section{Further Analysis of Energy Model}

\subsection{Comparison of Energy Consumption}

Figure 2 gives the energy consumption of multi-hop transmission, namely $E_{2}(n)$ in formula (7). Also, the energy consumption of direct transmission is included, which is equal to $E_{2}(1)$. Once again, we can easily see that $E_{2}(1)=E_{2}\left(n_{e}\right)$ and $\operatorname{Min}\left(E_{2}(n)\right)=E_{2}\left(n^{*}\right)=$ $E_{2}\left(\sqrt{n_{e}}\right)$. This gives us a judging criterion of choosing direct transmission or multi-hop transmission. In Section 4.2, we will present the numerical illustration and explanation.

Taking $n_{e}=100$ as an example, it will consume more energy when the real hop number increase from $n^{*}=\sqrt{n_{e}}=10$ to 100 . And it will consume less energy when real hop number increase from 1 to 10 . So, in the real sensor networks, we would prefer to transfer the data through 10 hops to the BS rather than 5 hops or 15 hops. Also, it is worth noting that since the real sensors are not placed with equal interval, the performance of real situation will always be worse than the ideal one.

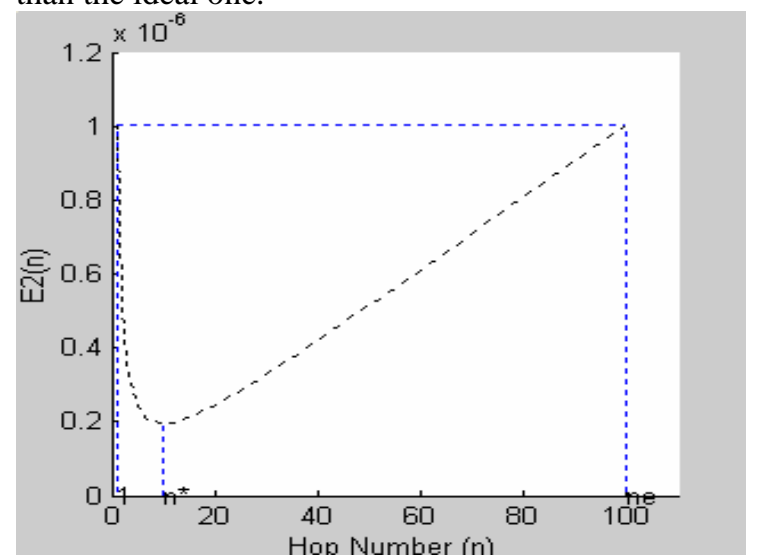

Figure 2. Comparison of energy consumption

\subsection{Influence of Design Parameters}

Intuitively, the design parameters in Table 2 will have a direct influence on the energy consumption model. Usually, they are not fixed and they are determined by the specific hardware components. For example, $E_{\text {elec }}$ varies from $10 \mathrm{~nJ} / \mathrm{bit}$ to 100 $n J$ / bit in [4]. Here, we also pick $E_{\text {elec }}$ as a variable and let the others be fixed value. Also, we can do it in a similar way as for the other design parameter.

As is shown in Table 3 , the fixed values are $\varepsilon_{\text {amp }}=100 \mathrm{pJ} / \mathrm{bit} / \mathrm{m}^{2}, E_{\text {elec }} \in[5,500] \mathrm{nJ} / \mathrm{bit}$, $d=100 \mathrm{~m}, l=1$ bit. Consequently, we can calculate $n_{e}$ according to formula (5).

Table 3. Different cases of design parameters

\begin{tabular}{|c|c|c|c|c|}
\hline $\begin{array}{c}E_{\text {elec }} \\
(\mathrm{nJ} / \mathrm{bit})\end{array}$ & $\begin{array}{c}\varepsilon_{\text {amp }} \\
\left(\mathrm{pJ} / \mathrm{bit}_{\mathrm{m}} \mathrm{m}^{2}\right)\end{array}$ & $\begin{array}{c}d \\
(\mathrm{~m})\end{array}$ & $\begin{array}{c}l \\
(\mathrm{bit})\end{array}$ & $n_{e}$ \\
\hline Case 1: 5 & 100 & 100 & 1 & 100 \\
\hline Case 2: 50 & 100 & 100 & 1 & 10 \\
\hline Case 3: 500 & 100 & 100 & 1 & 1 \\
\hline
\end{tabular}

It is interesting to see that, for Case 1, when sensor nodes are equally placed along a line from the source node to the BS, direct transmission consumes the same amount of energy as 100 multi-hop transmissions. In the mean time, there exists an optimal multi-hop number when the energy consumption is minimal during the communication session, and it is $n^{*}=\sqrt{n_{e}}=10$. Recall formula (1)-(3), here, the part of energy spent to run the radio is neglectable comparing to the part spent to run the amplifier since $E_{\text {elec }}$ is very small. Because $(n * r)^{2}>>n^{*} r^{2}$ (here $n=100, r=1$ ), it would take as many as 100 hops to run the radio so as to consume the same amount of energy as direct transmission. When the practical hop number is larger than $n_{e}$, it will consume more energy than $E_{2}\left(n_{e}\right)$, as is shown in Figure 2.

When $E_{\text {elec }}$ becomes larger in Case 2, the part of energy spent to run the radio plays a more important role. Consequently, it requires less multi-hop to run the radio so as to equalize $E_{2}(1)$ and $E_{2}\left(n_{e}\right)$. Still, there exists an optimal hop number $n^{*}$ to minimize the whole energy consumption. 
In Case 3, when $E_{\text {elec }}$ is large enough, it would be more economical to transmit the collected raw data through direct transmission rather than multi-hop transmission. The reason can be seen from formula (1)-(3), since here the energy spent to run the radio plays a dominating part and it would not be economical to use multi-hop transmission. From formula (4), we can also see that the inequation always holds true. Namely, direct communication will always consume less energy than multi-hop transmission, given the specific design parameters. In this case, there is no optimal multi-hop number.

\subsection{Judging Criterion of Transmission Manner}

Table 4 gives us a judging criterion when to choose direct transmission and when to choose multi-hop transmission.

Table 4. Judging criterion of transmission manner

\begin{tabular}{|c|c|c|}
\hline$n_{e}$ & $\begin{array}{c}\text { Direct } \\
\text { Transmission }\end{array}$ & $\begin{array}{c}\text { Multi-hop } \\
\text { Transmission }\end{array}$ \\
\hline$(0,2]$ & $\sqrt{ }$ & \\
\hline$(2,+\infty)$ & & $\sqrt{ }$ \\
\hline
\end{tabular}

When $n_{e} \in(0,1)$, it is similar to the Case 3 in Section 4.2. So, the source node will transmit its raw data directly to the BS. When $n_{e} \in[1,2], n^{*}$ is in the range of $[1, \sqrt{2}]$. Since $n^{*}$ is an integer, $n^{*}$ will be equal to 1 or 2 in the real situation. So, it is equivalent to direct transmission. When $n_{e}$ is larger than 2, $n^{*} \in(\sqrt{2},+\infty)$ and $n^{*}$ has a chance to choose any integer which is larger or equal to 2 . In this case, multi-hop transmission will consume less energy than direct transmission and there exists an optimal hop number $n^{*}$.

Up to now, we have solved the problem stated in Section 2.4. Once the design parameters are given and the distance from source node to the BS is known, we can select transmission manner according to the judging criterion listed in Table 4. To our knowledge, this aspect of work has not yet been comprehensively studied and it is our hope that this paper could be of some help to the practical engineering problem.

\section{An Energy Efficient Routing Scenario}

In this part, we will study an energy efficient routing scenario under a real network environment, rather than an ideal linear network environment. The problems are the same as those mentioned in Section 2.4.

Now, let us consider a wireless sensor network with $\mathrm{N}$ stationary nodes randomly deployed within a range of $[\mathrm{X}, \mathrm{Y}]$ area, as is depicted in Table 1 . When a source node has data to send, it will perform the following steps, as is depicted in Figure 3:

Step 1: Source node sends routing request (REQ) message through multi-hop routing until the message reaches the $\mathrm{BS}$; the REQ message includes all the intermediate nodes it travels through and the distance between each other.

Step 2: Once BS receives enough REQ(i),

Step 2.1: It first gets the distance from the source node to it since BS is location-aware, as is mentioned in the basic assumptions.

Step 2.2: It determines whether to choose direct transmission or multi-hop transmission according to Table 4.

Step 2.3: If through multi-hop transmission, it will decide the best REQ message from many candidate REQ messages according our strategy below.

Step 3: Once the best candidate is chosen, the corresponding intermediate nodes along the route are also decided and a reply (REP) message is sent back to the source node.

Step 4: Finally, the source node will know the route that raw data will pass through.

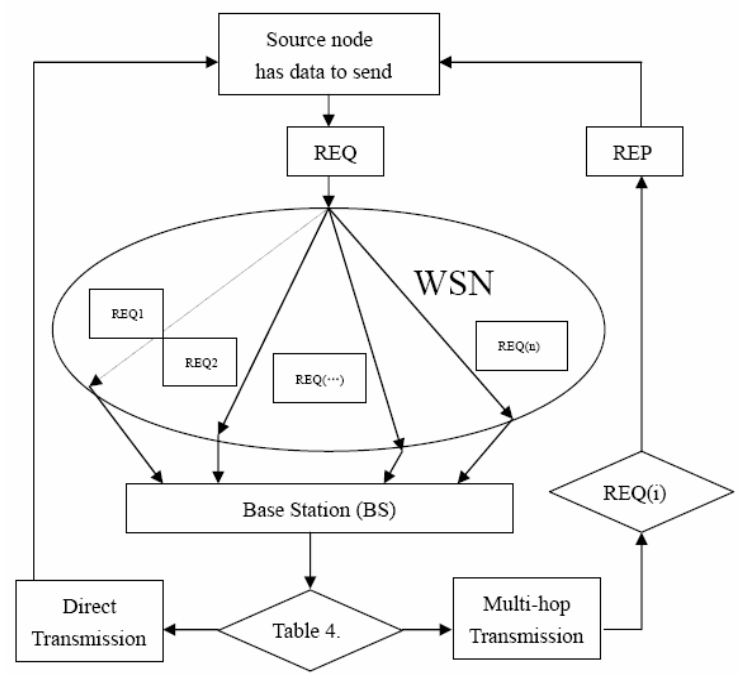

Figure 3. Diagram of our routing scenario

Since BS is powerful, most of the work is done by BS and only the decision is sent back to the source node. As we mentioned in Step 2.3, our strategy to choose the best candidate route so as to prolong the network lifetime is based on the following rules: 
Rule 1: BS will choose the actual hop number which is equal to $n^{*}, n^{*} \pm 1, n^{*} \pm 2 \ldots$

Rule 2: The variance of remaining energy should be as small as possible.

Rule 3: The average value of remaining energy should be as large as possible.

Rule 4: The priority is that Rule $2>1>3$.

Besides the average of remaining energy (Rule 3 ), we introduced the variance of remaining energy (Rule 2) as an important performance metric here. Our goal is to make balance of energy consumption among all of the sensors and we do not want to sacrifice some special nodes. We hope all the nodes will dissipate their energy at similar rate so that there will be no isolated area and the network lifetime can be prolonged as much as possible.

\section{Conclusions and Future Work}

In this paper, we make a comprehensive study about the relationship between hop number and energy consumption for Wireless Sensor Networks. After the analysis about energy consumption of different transmission manner and the influence of design parameters, we propose a transmission manner selection criterion, which is scalable and location unaware. Also, an energy efficient routing scenario with specific steps and diagram is presented to deepen the understanding of our method.

In the near future, we will perform simulation about energy consumption based on the scenario described above. Also we will concern scenarios of more Base Stations and BS at different positions. Last, but not the least important thing, we will make a comparison between our hop-based routing algorithm and other algorithms, such as shortest-hot algorithm, maximum remaining energy algorithm, in the aspects of hop number, network lifetime etc.

\section{Acknowledgements}

This research was supported by the MIC (Ministry of Information and Communication), Korea, under the ITFSIP (IT Foreign Specialist Inviting Program) supervised by the IITA (Institute of Information Technology Advancement) and was supported by the MIC (Ministry of Information and Communication), Korea, under the ITRC (Information Technology Research Center) support program supervised by the IITA (Institute of Information Technology Advancement) (IITA-2006-C1090-0602-0002)

\section{References}

[1] I.F. Akyildiz, W. Su, Y. Sankarasubramaniam, and E. Cayirci, "Wireless Sensor Networks: A Survey," Computer Networks, vol. 38, no. 4, 2002, pp. 393-422.

[2] R.C. Shah and J.M. Rabaey, "Energy Aware Routing for Low Energy Ad Hoc Sensor Networks," Proc. IEEE Wireless Comm. and Networking Conf., March 2002, pp. 350-355.

[3] C. Intanagonwiwat, R. Govindan and D. Estrin, "Directed diffusion: A scalable and robust communication paradigm for sensor networks", in the Proceedings of the 6th Annual ACM/IEEE International Conference on Mobile Computing and Networking (MobiCom'00), Boston, MA, August 2000.

[4] W. Heinzelman, A. Chandrakasan, and H. Balakrishnan, "Energy-efficient communication protocol for wireless sensor networks," in the Proceeding of the Hawaii International Conference System Sciences, Hawaii, January 2000.

[5] S. Lindsey and C. S. Raghavendra, "PEGASIS: Power Efficient GAthering in Sensor Information Systems," in the Proceedings of the IEEE Aerospace Conference, Big Sky, Montana, March 2002.

[6] O. Younis and S. Fahmy, "Heed: A hybrid, energyefficient, distributed clustering approach for ad-hoc sensor networks," IEEE Transactions on Mobile Computing, vol. 3, no. 4, October-December 2004, pp. 366--379.

[7] V. Rodoplu and T.H. Ming, "Minimum energy mobile wireless networks," IEEE Journal of Selected Areas in Communications, Vol. 17, No. 8, 1999, pp. 1333-1344.

[8] L. Li and J. Y Halpern, "Minimum energy mobile wireless networks revisited," in the Proceedings of IEEE International Conference on Communications (ICC'01), Helsinki, Finland, June 2001.

[9] K. Akkaya and M. Younis, "A Survey of Routing Protocols in Wireless Sensor Networks," in the Elsevier Ad Hoc Network, Vol. 3(3), pp. 325-349, 2005.

[10] Ivan Stojmenovic and Xu Lin, "Power-aware Localized Routing in Wireless Networks," IEEE Transactions on Parallel and Distributed Systems, vol. 12, no. 11, November, 2001, pp.1121-1133. 